\title{
Detection of Partial Invisible Objects in Images using Histogram Equalization
}

\author{
Satbir Singh \\ Assistant Prof. \\ Shaheed Udham singh \\ College of Engg. \& Technology, \\ Tangori, Punjab
}

\author{
Abhishek Godara \\ Assistant Prof. \\ Shaheed Udham singh College \\ of Engg. \& Technology, \\ Tangori, Punjab
}

\author{
Gaurav \\ Assistant. Prof. \\ Shaheed Udham singh College \\ of Engg. \& Technology, \\ Tangori, Punjab
}

\begin{abstract}
The current paper proposes recognition of partially invisible objects in images using image enhancement techniques. The problem mainly arises in night vision images which comprise poor contrast standards. Also during daytime, the object which is captured under sunlight is the lone survivor and the rest of information is not captured by camera properly. Image enhancement techniques to improve visual quality have been popularized with the proliferation of digital imagery and computers. Histogram Equalization (HE) is a versatile image improvement technique that can be incorporated for converting the partial visible objects/invisible objects into a proper vision. Further for enriching the information in image obtained by the HE image, a Contrast Limited Adaptive Histogram Equalization (CLAHE) is incorporated and finally for smoothing purpose, the image thus obtained is passed through a Gaussian filter. Results on various set of images show that above two techniques HE and CLAHE along with a Gaussian filter significantly improve the quality of image and hence assist to discover the partially visible/invisible objects.
\end{abstract}

\section{Keywords}

Object Detection, Invisible objects, Image Enhancement, Histogram Equalization (HE) and CLAHE.

\section{INTRODUCTION}

Detection of objects is a very interesting part of human life. Detecting cars, trees, buildings, people etc in a normal image is very easy. Problem occurs when image is captured in night with flash. Sometimes, while capturing images using cameras with flash in night only some portion of image becomes visible. By seeing through eyes one can only see the bright portion of the image and rest of the important information is missed [1]

For enhancing the dark portions of image, techniques based on histogram are useful. Some of the advantages of histogram based techniques i.e. Histogram Equalization are that they are less expensive comparing to other methods and provide simple approach for implementation of algorithms. This method is useful for images which are both dark and bright in backgrounds and foregrounds. There is a disadvantage that this method may increase the contrast of background noise and decrease the information signal.

There is another technique known as contrast limited adaptive histogram equalization (CLAHE), which processes small regions of image (known as tiles) using histogram specification for each tile individually. Neighboring tiles are then combined using bilinear interpolation to eliminate artificially induced boundaries. The contrast can be limited to avoid amplifying noise [2].
Further, additional filtering process using Gaussian filter is used to smoothen the image obtained after contrast limited adaptive histogram equalization (CLAHE) [2].

In this paper, two approaches namely HE and CLAHE are discussed for detection of partially visible objects on the images captured in dark environment especially in night and then filtered the equalized images using Gaussian filter.

The rest of this paper is organized as follows. In section 2, Histogram (HE and CLAHE) and introduction to Gaussian filter is discussed in detail with limitations and advantages. In section 3 proposed methodology is discussed. . In section 4 Results are discussed and finally conclusion and future scope is presented by section 5 .

\section{HISTOGRAM}

Generally, a Histogram of an image gives us a graphical representation of the tonal distribution of the gray code values in an image. With the help of Histogram frequency of appearing of different gray levels in an image is analyzed. Histogram was first introduced by Karl Pearson [3].

The histogram of a digital image with $\mathrm{L}$ total possible intensity levels in the range $[0, \mathrm{G}]$ is defined as the discrete function

$$
\mathrm{h}\left(\mathrm{r}_{\mathrm{k}}\right)=\mathrm{n}_{\mathrm{k}}
$$

where, $r_{k}$ is the $k^{\text {th }}$ intensity level in the interval $[0, G]$ and $n_{k}$ is the number of pixels in the image whose intensity level is $r_{k}$ . Also, $\mathrm{G}=\mathrm{L}-1$ for images of class uint8 and uint16 [2].

\subsection{Histogram Equalization (HE)}

Histogram Equalization is a technique which enhances the global contrast of an image. This technique is very useful especially when the usable information data of the image is represented by close contrast values. With this adjustment, the intensities can be better distributed on Histograms. Hence HE flattens the density distribution of the resulting image. HE is useful in images with more bright or darker backgrounds or images clicked in night with flash. Moreover this technique can lead to better views of bone structure in $\mathrm{x}$-ray images. The main advantage of this technique is that if the histogram equalization function is known, then original histogram can be recovered using the function. The limitation of this technique is that it may increase the contrast of the background noise, while decreasing the information signal. In spite of having advantages like contrast enhancement, high performance this technique is very rarely useful in televisions, since its straight use can change the original brightness of input image and deteriorate visual quality[2] [4].

Suppose that intensity levels in an image are continuous quantities normalized to range [0 1 l]. And let $\mathrm{p}(\mathrm{x})$ is the probability density function (PDF) of the intensity levels where $\mathrm{x}$ denotes the intensity value of normalized image. The 
density function of intensity distribution of the output image is equal to 1 due to histogram equalization, According to histogram equalization:

$$
\mathrm{Y}=\int_{0}^{x} \mathrm{p}(\mathrm{u}) \mathrm{du}
$$

Where $y$ is the intensity of output image, $x$ is the intensity of input image and $\mathrm{u}$ is a dummy variable. Both $\mathrm{x}$ and $\mathrm{y}$ lies in range [0 1]. [5]

\section{Mathematical expression:}

Let $X=\{X(i, j)\}$ denote a given image composed of $L$ discrete gray levels denoted as $\left\{\mathrm{X}_{0}, \mathrm{X}_{1} \ldots \ldots \mathrm{X}_{\mathrm{L}-1}\right\}$, where $\mathrm{X}(\mathrm{i}, \mathrm{j})$ represents an intensity of the image at the spatial location $(i, j)$.For a given image $\mathbf{X}$, the probability density function $\mathrm{p}\left(\mathrm{X}_{\mathrm{k}}\right)$ is defined as

$$
\mathrm{p}\left(\mathrm{X}_{\mathrm{k}}\right)=\mathrm{n}^{\mathrm{k}} / \mathrm{n}
$$

for $\mathbf{k}=0,1, \ldots, \mathrm{L}-1$, where $\mathrm{n}^{\mathrm{k}}$ represents the number of times that the level $X_{k}$ appears in the input image $X$ and $n$ is the total number of samples in the input image. Based on the probability density function, we define the cumulative density function as

$$
\mathrm{c}(\mathrm{x})=\sum_{j=0}^{k} \mathrm{p}\left(\mathrm{X}_{\mathrm{k}}\right)
$$

where $X_{k}=x$, for $k=0,1, \ldots, L-1 .[4]$

There are different types of histogram equalization techniques like Histogram expansion, Local Area Histogram Equalization (LAHE), Cumulative Histogram Equalization, Par sectioning, Odd sectioning, CLAHE . Histogram expansion is a simple technique of enhancing the contrast of image but it if there are grey values that are physically far apart from each other, then this technique will not be applicable. LAHE is similar to Histogram expansion in case of contrast enhancement but it is computationally very slow and requires high number of operations per pixel. Par sectioning is implemented easily but suitable for hardware implementation and odd sectioning offers good contrast but has problems with histogram covering full grey scale [6].

\subsection{CLAHE}

Contrast Limited Adaptive Histogram Equalization (CLAHE) is an improved version of Adaptive Histogram Equalization which overcomes the limitations of standard Histogram Equalization when grayscale distribution is highly localized and is not desirable to transform very low contrast images.. CLAHE algorithm divides the image into small contextual regions (tiles) and applies histogram equalization to each one. This technique was originally developed for medical imaging systems but it has proven to be successful for enhancement of low contrast images [7].

There are different parameters which are used in CLAHE. These are NumTiles, ClipLimit, Nbins, range, distribution and alpha. NumTiles is a 2 element vector of positive integers specifying the number of tiles by row and column. ClipLimit is a scalar parameter in range [ $\left[\begin{array}{ll}0 & 1\end{array}\right]$ which specifies the contrast enhancement limit. Nbins specify the number of bins for histogram used in building a contrast enhancing transformation. Range specifies the range of output image data. Distribution parameter is a string which specifies the desired histogram shape for the image tiles. Rayleigh, Uniform, exponential are the distribution parameters. Alpha is a nonnegative scalar parameter which is applicable to Rayleigh and exponential distributions [2].

\subsection{Introduction to Gaussian Filter}

Images are often corrupted by random variations in intensity, illumination, or have poor contrast and can't be used directly. A Gaussian filter is a linear low pass filter whose impulse response is a Gaussian function (or an approximation to it). Gaussian filters have the properties of having no overshoot to a step function input while minimizing the rise and fall time. The one dimensional Gaussian filter has an impulse response $\mathrm{g}(\mathrm{x})$ is given by

$$
g(x)=\frac{1}{2 \pi \sigma^{2}} e^{-\frac{x^{2}}{2 \sigma^{2}}}
$$

Where $\sigma$ is the standard deviation for the Gaussian kernel.

And in two dimensions,

$$
g(x, y)=\frac{1}{2 \pi \sigma^{2}} e^{-\frac{x^{2}+y^{2}}{2 \sigma^{2}}}
$$

Gaussian smoothing is very effective for removing Gaussian noise. The weights give higher significance to pixels near the edge (reduces edge blurring). Rotationally symmetric (perform the same in all directions). The degree of smoothing is controlled by $\sigma$ (larger $\sigma$ for more intensive smoothing) $[8$, $9,10]$.

\section{METHODOLOGY}

The job for enhancement of image for detection of partial invisible objects using our methodology involves the following basic steps:
(i) Capturing Input image
(ii) Histogram Equalization (HE)
(iii) Object Detection
(iv) Contrast Limited Adaptive Histogram Equalization (CLAHE)
(v) Filtering : Gaussian Filter

The proposed block diagram is shown in figure 1. Now, the methodology is discussed in detail.

First the input original image is converted into grayscale counterpart.

Then the histogram of above image is equalized to obtain a new image, which is richer in terms of the visual information about the image. This equalization can be done with different bin levels. 


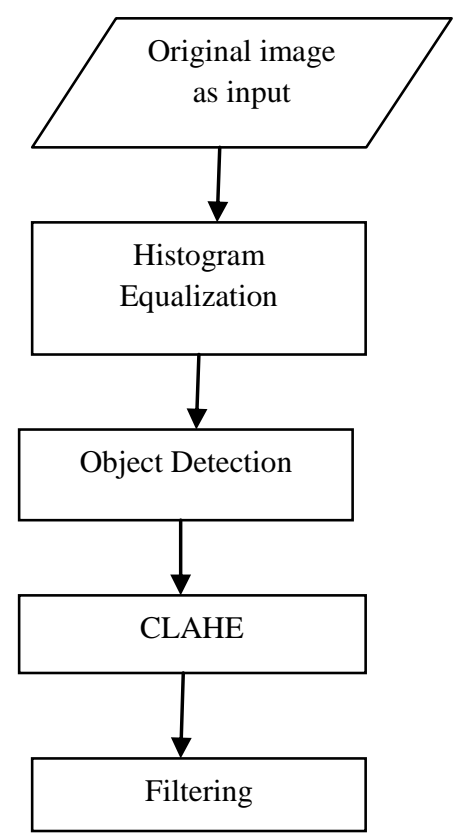

Figure1. Proposed Block diagram

Once we have obtained the equalized image, our aim is to detect the additional information it has provided as compared to the original image. The newly appeared objects/information is detected by simply using change detection.

Region marking can be done by choosing a certain region size and mark the objects which have area greater than a particular region/object size chosen.

After that for enhanced information and improving the contrast level, (since mainly used for images flashed during night vision) CLAHE is used.

Filtering refers to the selective processing of an image to remove unwanted aspects of the image or to transform only certain portions of the image. The main aim of employing additional Gaussian filter is to smooth the image and remove the blur effect from image.

\section{SIMULATION RESULTS}

Figure 2(a) shows the original image which is dark giving approximately no information, but when this image is equalized by histogram equalization as in (b), a lot of information is being fetched. Here a calendar, a PCB having different components is being identified. The red rectangles are used for highlighting the invisible objects. After HE, CLAHE is done to enhance the contrast of the image as shown in (c). Now, image after CLAHE may contain some noise which is filtered and smoothen by Gaussian filter with mean $=7$ and deviation $=10$. Figure $3(\mathrm{a})$ shows the histogram of the original image, (b) shows the histogram of equalized image. Figures 3(c) and (d) clearly show the difference between the histograms of the CLAHE and soothed filtered image.

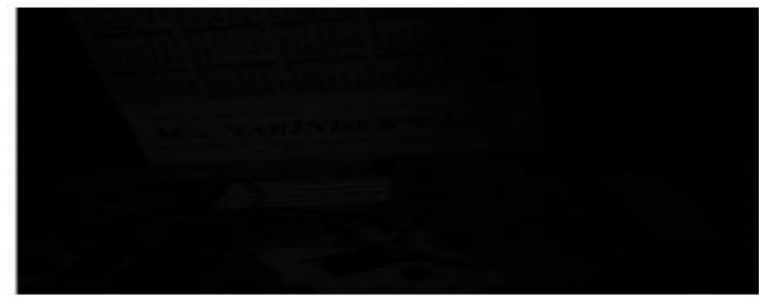

(a)

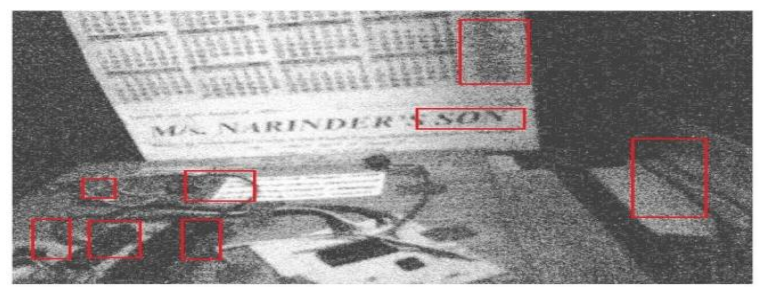

(b)

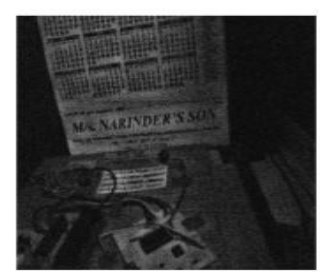

(c)

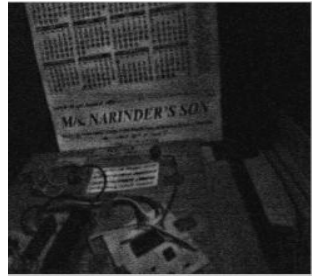

(d)
Figure 2. (a) Original Image. (b) Image after Histogram equalization. (c) Image after CLAHE. (d) Filtered Image

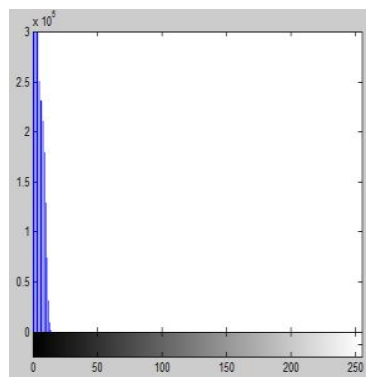

(a)

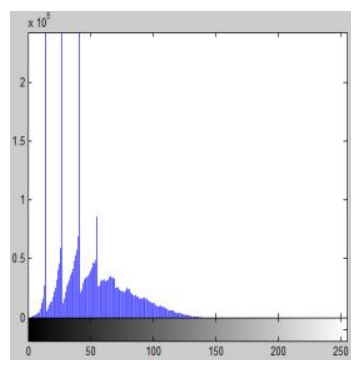

(c)

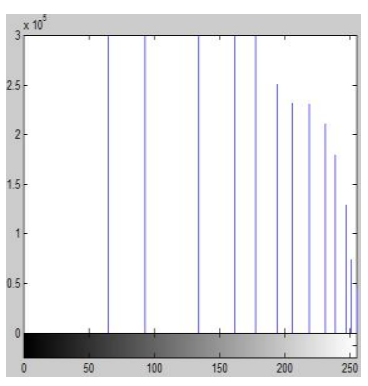

(b)

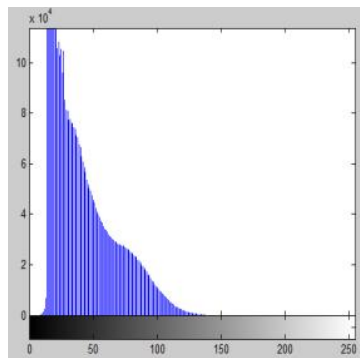

(d)
Figure 3 (a) Histogram of original image (b) Histogram of Equalized image (c) Histogram of CLAHE image (d) Histogram of Filtered image. 
Figure 4(a) shows the original image captured in night. In this image only the portion where the flash is more is visible (primary portion) rest of the portion remain dark. So, after using histogram equalization on this image, as shown in (b) the dark portions of the original image are detected. As compared to the original image, here a lady behind the man (primary portion), a towel, hole in the wall and legs of a person sleeping behind which were not visible in the original image are highlighted by using red rectangle.

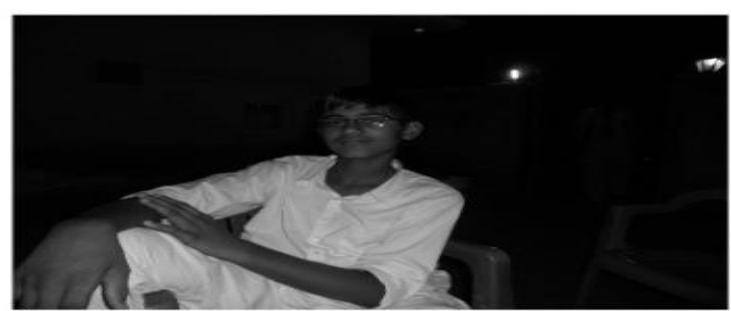

(a)

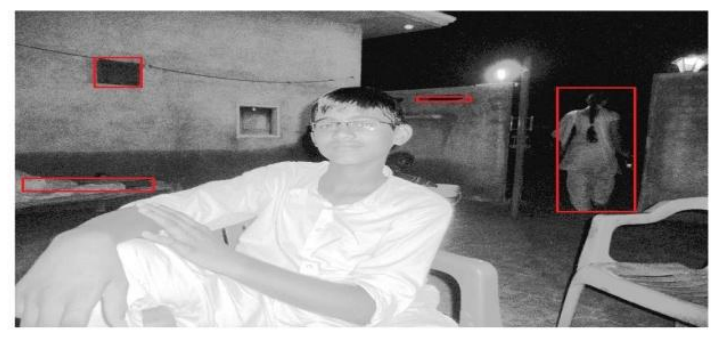

(b)

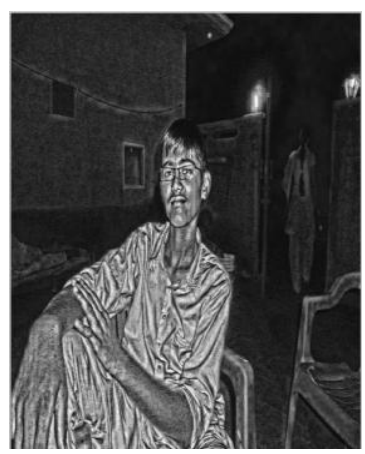

(c)

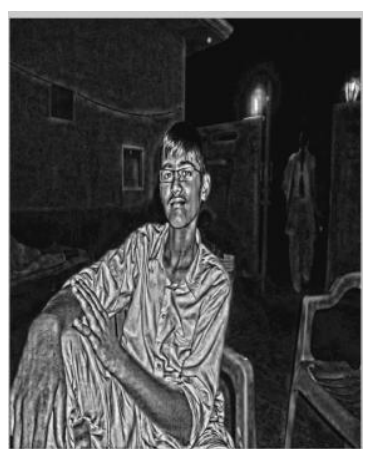

(d)
Figure 4. (a) Original Image. (b) Image after Histogram equalization. (c) Image after CLAHE. (d) Filtered Image.

After HE, in (c) contrast is improved thus bringing out more details of the image. Now, image after CLAHE may contain some noise which is filtered and smoothen by Gaussian filter with mean $=7$ and deviation $=10$.
Figure 5(a) shows the original image captured during day time but with a improper flash. Here though objects are partially visible but this image is not providing significant information regarding the objects. As shown in figure 2(b) the lady with a bag strip, person standing in front of the lady and three other such regions are highlighted via red rectangles. Rest CLAHE and filter application are same as in previous case with mean $=7$ and deviation $=10$.

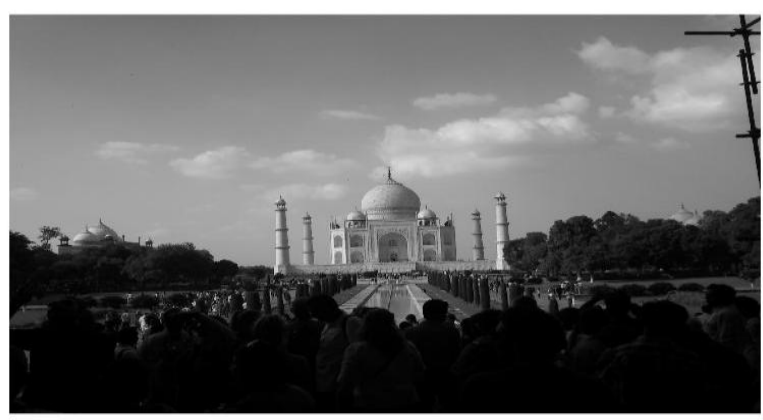

(a)

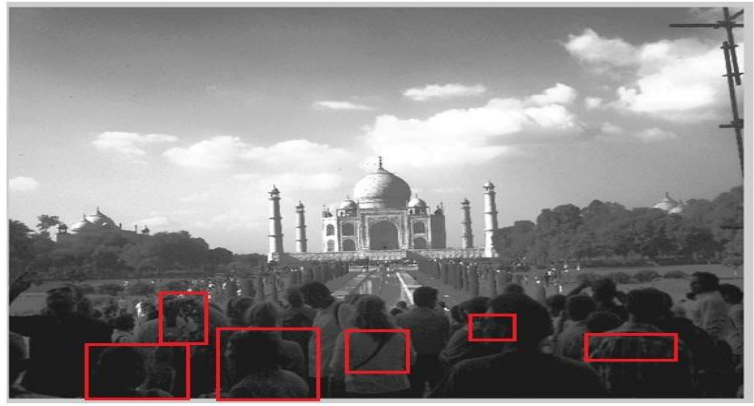

(b)

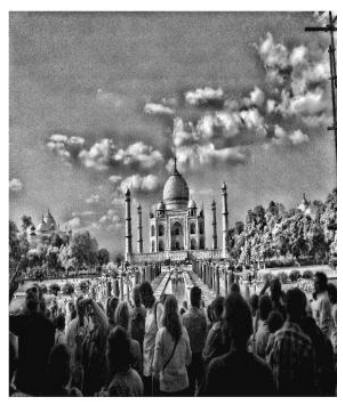

(c)

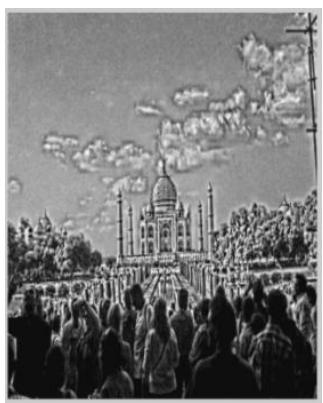

(d)
Figure5. (a) Original Image. (b) Image after Histogram equalization. (c) Image after CLAHE. (d) Filtered Image 


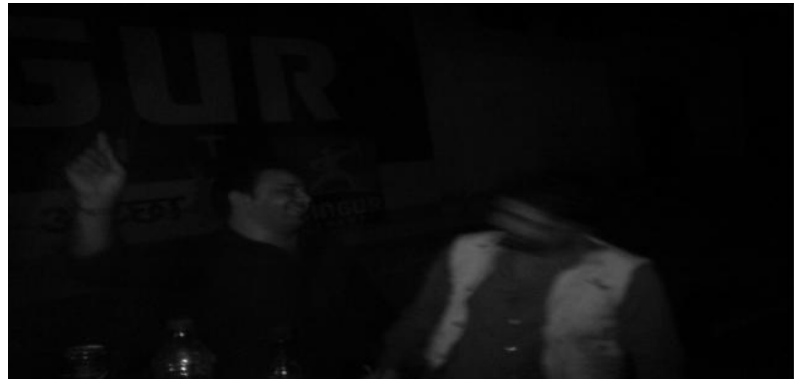

(a)

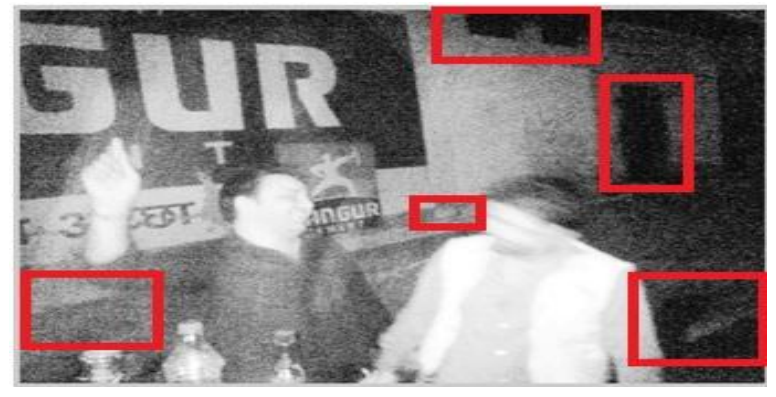

(b)

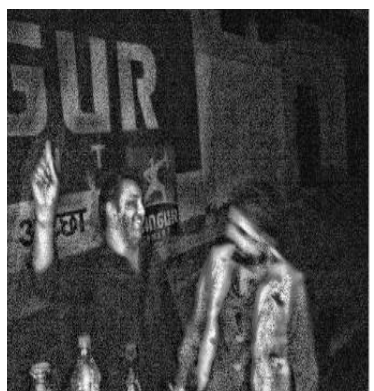

(c)

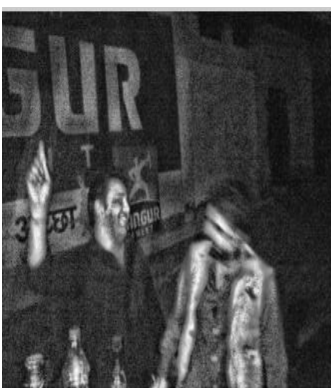

(d)
Figure6. (a) Original Image. (b) Image after Histogram equalization. (c) Image after CLAHE. (d) Filtered Image

Figure 6(a) showing the original image captured in night After histogram equalization as shown by (b) many objects have been identified (a door, two windows above the door etc.) which are highlighted by red rectangles. Rest CLAHE and filter application are same as in previous case with mean $=7$ and deviation $=10$.

The above results demonstrates that even in night vision (in absence of flash) or in day time (lack of brightness), good object detection is possible by using histogram equalization.

\section{CONCLUSION}

Detection of objects is a very remarkable part of human life. The difference in radiation intensity levels (both emitted and reflected) from a scene creates the information contained within an image. An object of interest can be identified by its contrast against its immediate surroundings, which defines the object's boundaries and edges. Problem occurs when image is captured in night with flash. By seeing through eyes one can only see the bright portion of the image and rest of the important information is missed. In this paper, detection of missed objects has been proposed using HE, CLAHE. HE is a versatile image enhancement tool mainly used in equalizing the contrast values across the whole image. Further to tackle the adverse conditions of extremely dark or bright scenario, a CLAHE can be incorporated to adjust the values adaptively by varying its different parameters like clip limit, range, distribution etc. This further enriches the contents of the recovered image. To gain out proper vision after CLAHE, an additional filtering process using a Gaussian filter is employed which helps in smoothing the image after the main missing objects were identified to remove any unwanted blur noise present in the image. The results were verified using many image sets, few of which are shown in the paper. In Figure2, small IC and electronic components which were earlier missing in the captured images were detected using the algorithms employed and marked using the red rectangles. This detection can be made using a particular region size depending upon the nature and size of objects present in the images. Some text portion was also recovered in the same image. Similarly in the second image, a lady and other objects not visible in the original image were highlighted in the enhanced image. Two more image sets were also shown which again clearly depicts the effectiveness of the proposed work.

\section{ACKNOWLEDGMENT}

The authors wish thanks to all who were directly or indirectly provide their kind support. We would also especially grateful to Mr. Harbinder Singh for providing necessary facilities to incorporate this research work.

\section{REFERENCES}

[1] Zhang Yu, Wang Xiqin, Peng Yingning, New Image Enhancement Algorithm for Night vision, IEEE.

[2] Rafael C. Gonzalez, Richard E. Woods, Steven L. Eddins. 2010 Digital Image processing. Tata McGraw Hill Education Pvt. Ltd

[3] Pearson, K. (1895). "Contributions to the Mathematical Theory of Evolution. II. Skew Variation in Homogeneous Material". Philosophical Transactions of the Royal Society A: Mathematical, Physical and Engineering Sciences 186: 343-414.

[4] Yeong Taeg Kim, 1997 Contrast Enhancement using Brightness Preserving Bi-Histogram Equalization, member, IEEE.

[5] H D Cheng, X J Shi 2004 A simple and effective Histogram Equalization approach to image enhancement. Science direct DSP 14(2004) 158-170,

[6] Robert Kruts, David Tenorio 2011, Histogram Equalization, Microcontrollers Solution group, Free scale semiconductor.

[7] Gibren Benitez-Garcia, Jesus Olivares -Mercado, Gualberto Aguilar-Torres, Gabriel Sanchez-Perez, Hector Perez-Meana, ,Face Identification based on contrast Limited Adaptive Histogram Equalization, Institute of Mexico.

[8] R.A. Haddad and A.N. Akansu, "A Class of Fast Gaussian Binomial Filters for Speech and Image Processing," IEEE Transactions on Acoustics, Speech and Signal Processing, vol. 39, pp 723-727, March 1991.

[9] Shapiro, L. G. \& Stockman, G. C: "Computer Vision", page 137, 150. Prentice Hall, 2001

[10] Mark S. Nixon and Alberto S. Aguado. Feature Extraction and Image Processing. Academic Press, 2008, p. 88. 